\title{
Thoracoscopic Resection of an Intercostal Neurinoma
}

\author{
Apostolos C. Agrafiotis ${ }^{\mathrm{a}}$, Polychronis Ikonomidis ${ }^{\mathrm{a}}$, Nikolaos Stamos ${ }^{\mathrm{a}}$, Sotirios Moraitis ${ }^{\mathrm{a}, \mathrm{b}}$
}

\begin{abstract}
Neurinomas are benign tumors representing up to $5 \%$ of soft tissue neoplasms, discovered mostly incidentally. We report the case of a 25-year old Caucasian male patient with a left apical "coin” lesion on a routine chest radiogram. Further radiologic workup and a percutaneous transthoracic biopsy revealed an intercostal neurinoma. A thoracoscopic resection of the tumor was performed. The pathologic examination confirmed the initial diagnosis. Follow-up after 12 months showed no tumor recurrence. The surgical technique is described and different aspects regarding diagnosis and treatment of intercostal neurinomas are further discussed.
\end{abstract}

Keywords: Thoracoscopy; Intercostal; Neurinoma

\section{Introduction}

Neurinomas are benign nerve sheath tumors, with no sex or age predilection. Intrathoracic neurinomas arising from intercostal nerves are rare and are discovered incidentally. Thoracoscopic resection is the treatment of choice for totally intrathoracic lesions. The case of a 25-year old Caucasian male patient with an intercostal neurinoma is reported. A thoracoscopic resection of the tumor was performed. This article's aim is to draw attention to this infrequent pathology, describe the surgical technique and to discuss different aspects regarding diagnosis and treatment of intercostal

Manuscript accepted for publication June 23, 2011

${ }^{a}$ Department of Thoracic Surgery, Athens Naval and Veterans Hospital, Athens, Greece

${ }^{\mathrm{b}}$ Corresponding author: Sotirios Moraitis, Department of Thoracic Surgery, Athens Naval and Veterans Hospital, 70 Dinokratous st., 115 21 Athens, Greece. Email: s_moraitis@hotmail.com

doi:10.4021/jmc243w neurinomas.

\section{Case Report}

The patient, one month before the referral to our department, had a routine chest radiogram, which revealed a left apical "coin” lesion (Fig. 1). The patient was asymptomatic and had no past medical history. A percutaneous computed tomography-guided biopsy set the diagnosis of a neurinoma. A magnetic resonance imaging (MRI) was performed and showed at the level of the left paravertebral region, an extrapleural mass with smooth edges arising from the second intercostal space (Fig. 2). Its diameter was $2.7 \mathrm{~cm}$. There was no communication with the spinal canal. After intravenous contrast injection there was an intense enhancement of the tumor (Fig. 3a, b). The overall imaging was highly suggestive of a peripheral nerve sheath tumor.

Laboratory findings and preoperative respiratory function tests were within normal limits.

The patient presented no other intrathoracic or cutaneous lesions suggestive of neurofibromatosis.

A thoracoscopic resection of the lesion was performed.

After the intubation with a double-lumen endotracheal

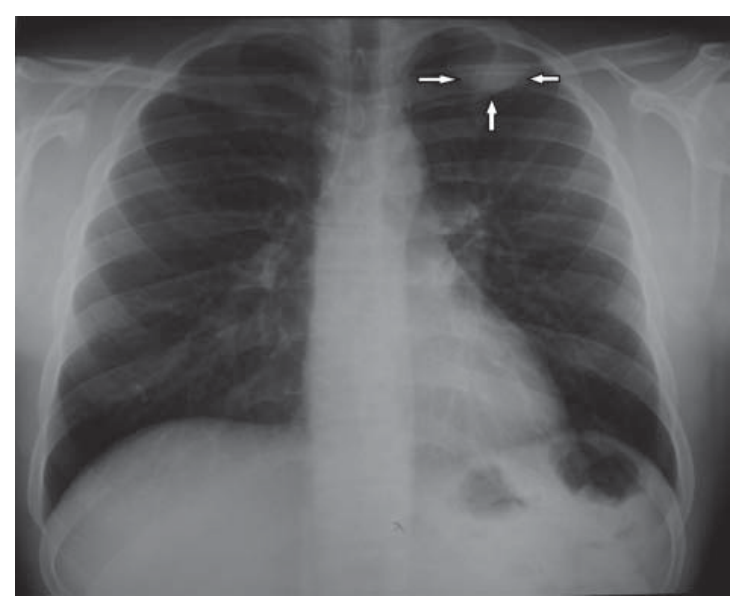

Figure 1. Chest radiogram showing a left apical "coin" lesion (arrows). 


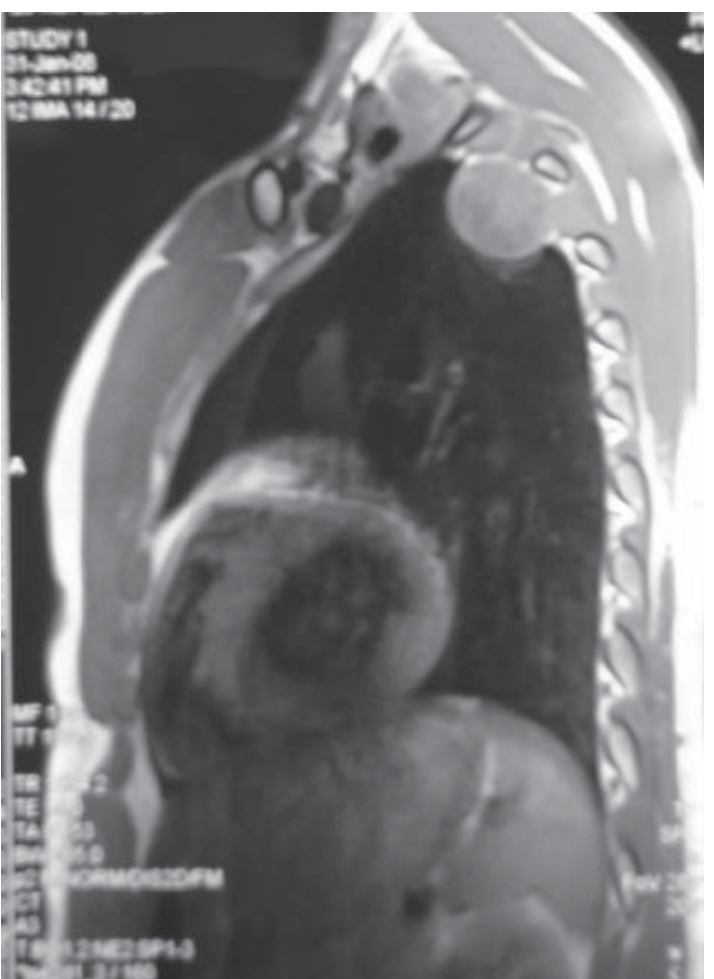

Figure 2. MRI image showing the mass arising from the second intercostal space.

tube for ipsilateral lung collapse, the patient was installed as for a standard posterolateral left thoracotomy.

In order to introduce the thoracoscope, a $12 \mathrm{~mm}$ thoracoscopic port was used and was placed on the seventh intercostal space in the mid-axillary line. A $30^{\circ}$ thoracoscope was used.

In addition, two $12 \mathrm{~mm}$ ports were used as working ports. They were placed on the third and fourth intercostal spaces at the level of the anterior and posterior axillary line respectively.

When the lesion was visualized, the parietal pleura was incised circumferentially around it, by using the electrocautery hook. There was a communication between the tumor and the second intercostal nerve by a small pedicle.

The tumor was easily dissected free from the surrounding tissues of the thoracic wall and its pedicle was directly electrocoagulated, while a double vascular clip was placed peripherally to the tumor.

An endoscopic bag was used in order to extract the tumor from the pleural cavity (Fig. 4a, b). The porthole at the level of the posterior axillary line was enlarged up to $5 \mathrm{~cm}$.

A chest tube was introduced under direct vision. The lung was reexpanded and the skin incisions were closed. The total operating time was $107 \mathrm{~min}$ and there was no significant blood loss.

The patient's postoperative course was uneventful. The chest tube was taken out on the third postoperative day. day.

The patient was discharged on the sixth postoperative

The pathologic examination confirmed the initial diagnosis of a neurinoma. There were no signs of malignancy.

One month after the operation the patient was seen in our outpatient clinic. Clinical examination was normal. The chest radiogram performed at that time did not present any pathology.

Twelve months postoperatively follow-up imaging showed no tumor recurrence.

\section{Discussion}

The peripheral nerve sheath tumors arise from cells of the peripheral nerve, including Schwann cells, perineurial cells and fibroblasts [1].

Benign nerve sheath tumors are subdivided into two separate morphological groups with different histopathological characteristics: schwannoma (also known as neurilemoma or neurinoma) and neurofibroma [2].

Neurinomas represent approximately $5 \%$ of benign softtissue neoplasms [2]. They can occur at all ages, though they are uncommon in children. They are most prevalent between the ages of 20 and 50, with no sex predilection. The majority of neurinomas occur sporadically in patients without a
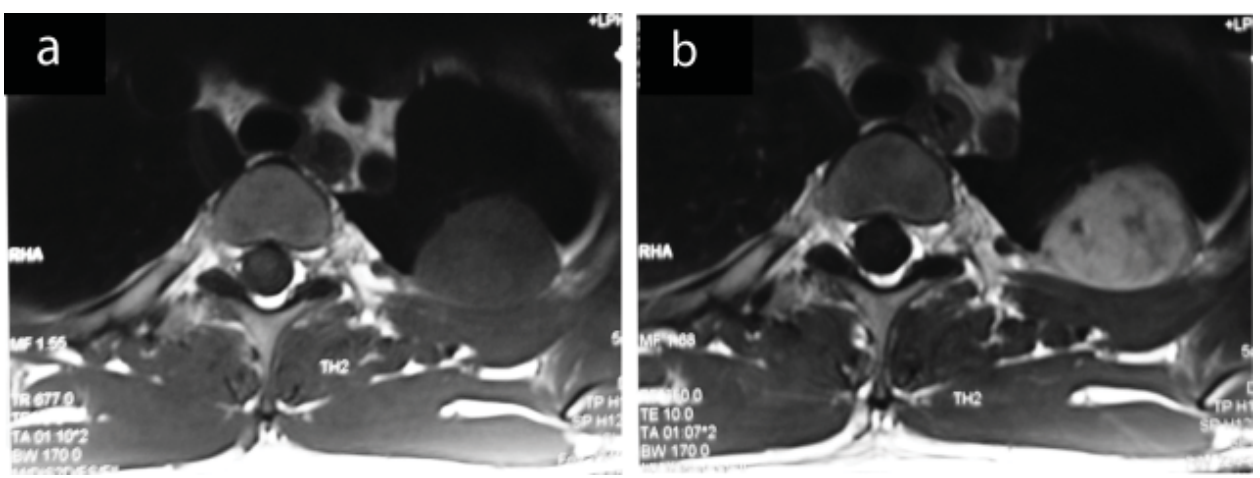

Figure 3. MRI images showing a marked enhancement of the mass after contrast injection $(a, b)$. 

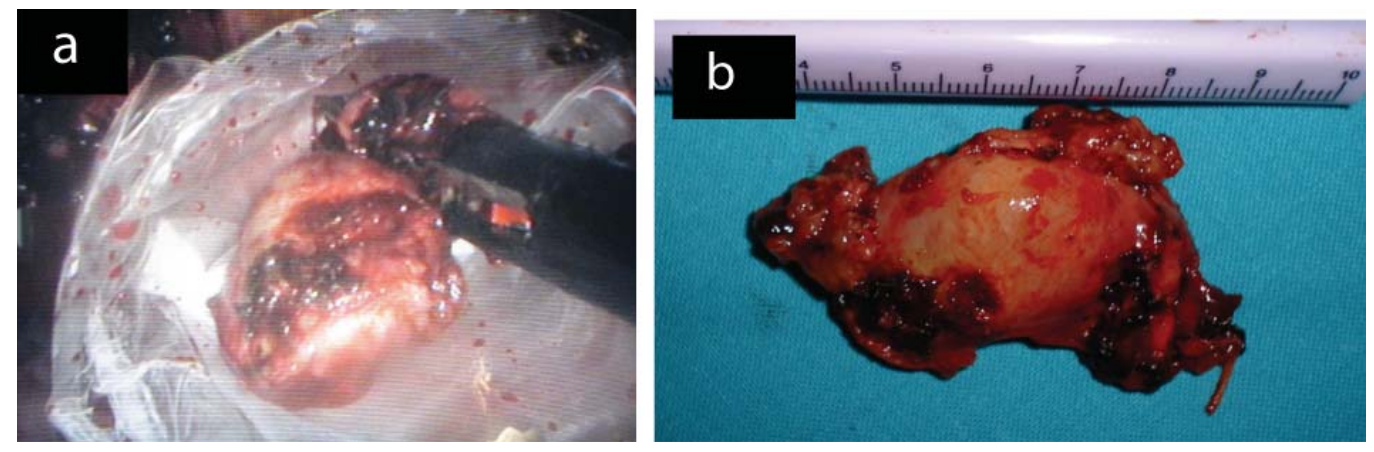

Figure 4. The extraction of the tumor in an endoscopic bag and the tumor after its extraction from the pleural cavity $(a, b)$.

genetic predisposition. However, histologically identical tumors also occur in patients with neurofibromatosis type 2, or schwannomatosis [2]. Malignant transformation of neurinomas is rare [3].

Neurinomas are usually asymptomatic and are incidentally discovered on imaging studies; however pain or other symptoms may occur due to compression of the involved nerve or adjacent structures. Horner syndrome, hemothorax, dyspnea and subcutaneous tumors of the chest wall are some rare presentations of neurinomas arising from intercostal nerves [3-5].

Rarely, rib notching can be seen on chest radiography in case of neurinomas of the intercostal nerves. Moreover, a posterior mediastinal mass, should suggest the possibility of a neurogenic tumor, for they account for $30 \%$ of posterior mediastinal tumors [2].

MRI is the preferred method for the assessment of peripheral nerve sheath tumors [6]. Vessels in neurinomas are usually prominent and their rich vascular supply is reflected in the often intense enhancement of these tumors on imaging studies [2].

Since on imaging studies neurinomas may mimick other pathologies including malignant lesions, a preoperative biopsy of the tumor, when feasible, is necessary in order to avoid overtreatment [7].

Peripheral nerve sheath tumors arising from intercostal nerves may be totally intrathoracic or they may have in addition an intraspinal expansion, as well (known as hourglass or dumbbel tumors) [8. 9].

For the latter a combined resection is suggested through thoracotomy or thoracoscopy and laminectomy in order to achieve total resection [8].

On the other hand, for the tumors that are limited into the thoracic cavity, thoracoscopic resection is advocated as the treatment of choice [9].

The neurinoma displaces normal elements of the nerve to one side; therefore surgical excision usually spares the parent nerve because the tumor can be easily separated from the underlying nerve fibers [2].

The patients benefit from all the advantages of a mini- mally invasive procedure (less operative stress, less postoperative pain, shorter hospitalisation, better cosmetic result etc.) as there is no muscle transection and rib retraction as in classic thoracotomy [10].

However, the operating surgeon should not hesitate to convert to a thoracotomy, in front of an uncontrollable hemorrhage, the risk of an incomplete resection or the possibility of malignancy [8]. Complete resection is essential even for benign lesions, as there is the risk of local recurrence [9].

The insertion sites for the thoracic ports and the choice of thoracoscope depends on the location of the tumor. A single injection of antibiotics must be given before port placement [11].

The dissection of the tumor can be performed either by using electrocoagulation or ultrasonic scalpel, paying attention not to grasp the tumor directly. Meticulous haemostasis at the level of the intercostal spaces is essential.

All specimens should be removed in a bag to avoid contamination of the chest cavity by tumor cells which could have serious consequences in case of unexpected malignancy. The extraction is performed through a limited "utility thoracotomy". For cosmetic reasons, the highest port hole incision in the axilla is used for that purpose, at least when feasible [11].

The placement of a chest tube at the end of the operation under direct vision is generally recommended.

The postoperative complications after a thoracoscopic resection of these lesions are usually minor and self-limited (transient intercostal neuralgia, paresthesias, pleural effusion etc.) $[10,12]$.

Follow-up imaging is recommended in order to exclude local recurrence.

In conclusion, neurinomas arising from intercostal nerves is a rare entity and are often discovered incidentally. Resection is generally recommended in order to confirm the preoperative diagnosis and to exclude the possibility of malignancy. When the tumors are totally intrathoracic, a thoracoscopic approach should be performed; however conversion to thoracotomy is mandatory in front of the risk of an incomplete resection or other perioperative difficulties and 
in case of suspected malignancy.

\section{Conflict of Interest}

The authors declare that there is no conflict of interest

\section{References}

1. Frosch MP, Antoni DC, De Girolami U. Peripheral nerve sheath tumors. In: Kumar V, Abbas A, Fausto N. Robbins and Cotran Pathologic Basis of Disease, 7th Edition. Philadelphia, Elsevier Saunders, 2005:1411-3.

2. Parizel PM, Geniets C. Tumors of peripheral nerves. In: DeSchepper AM, Vanhoenacker F, Gielen J, Parizel PM eds. Imaging of soft tissue tumors, 3rd edition. Berlin, Heidelberg, New York, Springer, 2006:325-53.

3. Sakurai H, Hada M, Mitsui T, Ashizawa I. Extrathoracic neurilemoma of the lateral chest wall mimicking a subcutaneous tumor: report of a case. Ann Thorac Cardiovasc Surg. 2006;12(2):133-136.

4. Martin-Ucar AE, Rengarajan A, Waller DA. Giant intercostal nerve Schwannoma presenting as Horner's syndrome. Recovery after surgical resection. Eur J Cardiothorac Surg. 2002;22(2):310.

5. Lee MH, Graham AN, Nicholson AG, Pastorino U. Soli- tary cellular schwannoma presenting with haemothorax. J R Soc Med. 1998;91(11):596-597.

6. Petteruti F, De Luca G, Lerro A, Luciano A, Cozzolino I, Pepino P. Intercostal ancient schwannoma mimicking an apical lung tumour. Thorax. 2008;63(9):845-846.

7. Urakawa T, Kawakita N, Nagahata Y. A case of benign schwannoma of the thoracic wall mimicking a malignant tumor. Kobe J Med Sci. 1993;39(4):123-131.

8. Okada D, Koizumi K, Haraguchi S, Hirata T, Hirai K, Mikami I, Fukushima M, et al. A case of dumbbell tumor of the superior mediastinum removed by combined thoracoscopic surgery. J Nihon Med Sch. 2002;69(1):58-61.

9. Nakamura H, Komagata M, Nishiyama M, Taguchi M, Kawasaki N. Resection of a dumbbell-shaped thoracic neurinoma by hemilaminectomy: a case report. Ann Thorac Cardiovasc Surg. 2007;13(1):36-39.

10. Han PP, Dickman CA. Thoracoscopic resection of thoracic neurogenic tumors. J Neurosurg. 2002;96(3 Suppl):304-308.

11. Valla J-S, Leclair MD, Heloury Y. Thoracoscopic removal of mediastinal neurogenic tumors. In: Bax KNMA, Georgeson KE, Rothenberg SS, Valla J-S, Yeung CK eds. Endoscopic Surgery in Infants and Children. Berlin, Heidelberg, New York, Springer, 2008:189-97.

12. Hazelrigg SR, Boley TM, Krasna MJ, Landreneau RJ, Yim AP. Thoracoscopic resection of posterior neurogenic tumors. Am Surg. 1999;65(12):1129-1133. 